\title{
Mean kinetic energy and final state effects in liquid hydrogens from inelastic neutron scattering
}

\author{
G.Corradi ${ }^{1,2}$, D.Colognesi ${ }^{1}$, M.Celli ${ }^{1}$, M.Zoppi ${ }^{1}$ \\ 1 Consiglio Nazionale delle Ricerche, \\ Istituto di Fisica Applicata 'Nello Carrara', \\ Via Panciatichi 64, 50127, Florence, Italy \\ 2 Università degli Studi di Firenze, Dipartimento di Fisica, \\ Via G. Sansone 1, 50019, Sesto Fiorentino (FI), Italy
}

Received April 29, 2003, in final form July 21, 2003

\begin{abstract}
We have measured, using the TOSCA spectrometer at ISIS, the neutron scattering spectrum of liquid para-hydrogen ( $T=19.3 \mathrm{~K}$ and $n=$ $\left.25.4 \mathrm{~nm}^{-3}\right)$ and liquid ortho-deuterium $\left(T=21.3 \mathrm{~K}\right.$ and $\left.n=25.2 \mathrm{~nm}^{-3}\right)$. From the high-energy region of the spectrum, namely $40 \mathrm{meV}<\hbar \omega<$ $1 \mathrm{eV}$, where the incoherent approximation for the center-of-mass motion applies, we have been able to extract the translational mean kinetic energy, which, as expected, turns out to be rather different from the classical values. However, significant deviations from the impulse approximation have been detected and the inclusion of some correction terms (accounting for the so-called final state effects) had to be accomplished in order to accurately describe the experimental spectra. The comparison of the present mean kinetic energy values with the available experimental and simulated data in the literature is quite good and confirms the excellent performances of TOSCA in the spectroscopic analysis of the condensed phases of liquid hydrogens.
\end{abstract}

Key words: liquid para-hydrogen, liquid ortho-deuterium, mean kinetic energy, final state effects

PACS: $67.90 .+z, 61.12 . E x$

\section{Introduction}

One of the important features of the liquid hydrogens (i.e. $\mathrm{H}_{2}$ and $\mathrm{D}_{2}$ ), among all the quantum liquids, is their intermediate character between a deeply quantum system like helium, where exchange effects can be relevant and characterize the system features, and a weakly quantum system like neon or methane, where quantum effects are relatively small and can be calculated using a perturbative approach 
applied to a classical reference model [1]. As a matter of fact, the quantum features of the hydrogens are known to be relevant [2], even though the presence of effects that could be ascribed to the particle exchange has never been experimentally detected in the liquid state. Therefore, $\mathrm{H}_{2}$ and $\mathrm{D}_{2}$ represent an important case where quantum exchange does not play an appreciable role, but the system structure and dynamics are still largely influenced by the quantum delocalization properties [3]. In other words, hydrogens are an effective practical example of a Boltzmann quantum liquid.

From a purely experimental point of view, dealing with a Boltzmann liquid is not very different from dealing with a classical or even a superfluid one. However, the difference emerges in its full glory at the time of interpreting the experimental results, when a comparison with the theoretical predictions is carried out. As far as the static properties are concerned, the state of the art of computer simulation has evolved to such an extent that almost all microscopic static properties of quantum liquids (including superfluid ${ }^{4} \mathrm{He}$, with the only possible exception of liquid ${ }^{3} \mathrm{He}$ at temperature $T>0[4]$ ) can be precisely evaluated using the Path Integral Monte Carlo (PIMC) technique [5]. However, even though some interesting and promising results have begun appearing in the literature [6-12], no simulation approach has been proved so far to give a complete and precise description of the dynamics of a quantum liquid. The microscopic dynamics in a Boltzmann liquid is also interesting if one takes into account that, from a theoretical point of view, this is still an open problem. Therefore, the possibility of carrying out experiments on real liquids that map almost exactly this model is of paramount importance, and the liquid hydrogens represent one of the few examples where the Boltzmann quantum liquid theory could be applied to real systems.

Quantum effects in Boltzmann liquids originate from the delocalization of particles whose center-of-mass (COM) positions cannot be associated with individual points in space, but are characterized by a probability distribution [13]. This, in turn, is somehow related to the de Broglie thermal wavelength, $\lambda_{\mathrm{DB}}$, which depends on the particle mass and the system temperature, according to the definition [14]:

$$
\lambda_{\mathrm{DB}}=h /\left(2 \pi M k_{\mathrm{B}} T\right)^{1 / 2},
$$

where $M$ is the particle mass, $T$ is the temperature, $h$ is the Planck constant, and $k_{\mathrm{B}}$ is the Boltzmann constant. Strictly speaking, the above definition of the de Broglie thermal wavelength is applicable to a massive free particle by identifying its momentum, $\mathbf{p}=M \mathbf{v}$, with the momentum of the associated plane wave, $\mathbf{p}=\hbar \mathbf{k}$, where $\mathbf{k}$ is the wave-vector. However, for a dense system of interacting particles a different definition should be used. Let us consider, for example, a single molecule in a dense liquid of identical (but still distinguishable, according to the Boltzmann statistics) particles. If we could take a snapshot of this particle, surrounded by the first shells of its neighbors, we would see that the space available to the tagged molecule is limited by the cage-effect produced by the nearest neighbors. This implies the existence of an upper limit for the fraction of the space, $\Delta \mathbf{r}$, available to the tagged-particle wave-function. However, due to the Heisenberg uncertainty principle, this has an implication on the minimum size of the momentum variance, $\Delta \mathbf{p}$. As a consequence, 
the mean kinetic energy associated to a single particle (which is determined by the squared momentum: $\left.\left\langle E_{k}\right\rangle=\mathbf{p}^{2} / 2 M\right)$ assumes a value that is larger than what is expected on the basis of the classical equipartition theorem (i.e. $\left.\left\langle E_{k}\right\rangle_{\mathrm{cl}}=(3 / 2) k_{\mathrm{B}} T\right)$. Furthermore, the intermolecular interaction details do not contribute very much to the mean kinetic energy value. This effect is generally observed at very low temperatures, below the liquid Debye temperature [15], and is somehow equivalent to the zero-point motion effect of the molecules composing a solid.

In order to be more rigorous, we introduce the momentum distribution of the single particle $n(\mathbf{p})$. Classically, this is given by the well-known Maxwell-Boltzmann distribution that is:

$$
n(\mathbf{p})=\left(\frac{\beta}{2 \pi M}\right)^{3 / 2} \exp \left(-\beta \frac{\mathbf{p}^{2}}{2 M}\right),
$$

where $\beta=1 / k_{\mathrm{B}} T$. The space-Fourier transform of the momentum distribution, namely:

$$
\tilde{n}(\mathbf{r})=\int \mathrm{d} \mathbf{p} \exp \left(-\frac{\mathrm{i}}{\hbar} \mathbf{p} \cdot \mathbf{r}\right) n(\mathbf{p})
$$

represents the one-body density matrix, i.e. the statistical average of the overlap between the system wave-function and itself, after shifting the coordinates of one particle by the quantity $\mathbf{r}$ (keeping the coordinates of all the other particles fixed). It is easy to show [16] that the one-body density matrix describes in a rigorous way the delocalization of a single particle in an interacting system, both caused by quantum and by thermal effects. We note that the momentum distribution is normalized in such a way that:

$$
\int \mathrm{d} \mathbf{p} n(\mathbf{p})=1
$$

which implies that the normalization of $\tilde{n}(\mathbf{r})$ is simply: $\tilde{n}(0)=1$. Starting from the Maxwell-Boltzmann distribution, one writes:

$$
\tilde{n}(\mathbf{r})=\exp \left(-\frac{M \mathbf{r}^{2}}{2 \beta \hbar^{2}}\right)
$$

which means that the standard deviation of the single-particle delocalization is:

$$
\sigma=\hbar /\left(M k_{\mathrm{B}} T\right)^{1 / 2},
$$

a result which is very similar to the definition of the de Broglie wavelength (see equation (1)). It is worthwhile to observe that, by this procedure, we have obtained a sort of a thermally averaged wave-packet, even for a classical particle obeying to the Maxwell-Boltzmann momentum distribution.

We have seen that in a classical system, $n(\mathbf{p})$ has a Gaussian shape, given by the Maxwell-Boltzmann distribution. However, it is known that the Gaussian shape is lost for a strongly quantum mechanical system, such as ${ }^{4} \mathrm{He}$ in the vicinity or below the $\lambda$-transition [17-22], where the emergence of a long-range tail in $\tilde{n}(\mathbf{r})$ is interpreted as a measure of the Bose-condensate fraction. However, looking at a less 
quantum system, like neon, no significant deviation from a Gaussian distribution has been clearly experimentally detected [23], while recent PIMC simulations indicate that the momentum distribution of neon maintains its Gaussian shape for several orders of magnitude, both in the liquid and the solid phase [24]. These results are also corroborated by the general theoretical finding [25] that the quantum corrections to a classical Maxwell-Boltzmann momentum distribution retain a Gaussian functional form up to the $\hbar^{2}$ perturbative order. This suggests that the validity range of a Gaussian momentum distribution could be extended to include Boltzmann quantum liquids too.

One might argue that quantum effects in neon momentum distribution are not so large to evidence deviations from the Gaussian shape. However, this hypothesis has been also successfully used to interpret deep inelastic neutron scattering data in liquid helium far from the $\lambda$-transition [26], and in dense supercritical ${ }^{4} \mathrm{He}$ [27]. By assuming that a Gaussian shape for the momentum distribution also applies to the Boltzmann quantum liquids, one can extrapolate the validity of equation (2) by simply replacing the classical value of the single-particle mean kinetic energy, i.e. $\left\langle E_{k}\right\rangle_{\mathrm{cl}}=(3 / 2) k_{\mathrm{B}} T$, with its actual quantum mechanical value, $\left\langle E_{k}\right\rangle$. Thus, equation (2) becomes:

$$
n(\mathbf{p})=\left(\frac{3}{4 \pi M\left\langle E_{k}\right\rangle}\right)^{3 / 2} \exp \left(-\frac{3 \mathbf{p}^{2}}{4 M\left\langle E_{k}\right\rangle}\right) .
$$

Consequently, the one-body density matrix (see equation (5)) writes:

$$
\tilde{n}(\mathbf{r})=\exp \left(-\frac{M\left\langle E_{k}\right\rangle \mathbf{r}^{2}}{3 \hbar^{2}}\right)
$$

Thus, in this framework, the knowledge of the real value of the quantum mechanical single-particle mean kinetic energy is sufficient to determine the single-particle momentum distribution and its Fourier transform, i.e. the one-body density matrix. We point out, once more, that this is only a working hypothesis which is mostly based on the properties of neon. It would be important to know if the same scenario applies to other Boltzmann liquids characterized by quantum effects of a larger size. More experimental data and simulations, carried out on liquid hydrogens, could be quite helpful in solving this problem.

\section{The experimental point of view}

Measuring the single-particle momentum distribution in a dense interacting system is not a trivial task by any means. However, it has been shown that this physical quantity can be accessed, in an almost direct way, by using high-energy neutrons $[28,29]$ in conjunction with a technique known as Deep Inelastic Neutron Scattering or Neutron Compton Scattering (NCS) [30].

As a matter of fact, if the energy of the neutron is much larger than any energy scale involved in the atomic system interactions (we remind that $5 \mathrm{eV}$ roughly 
correspond to $58.000 \mathrm{~K}$ ) the scattering process is not influenced by the local environment of the target atom after the scattering event. This is generally called short-time approximation or impulse approximation (IA) [30]. In addition, due to the large wave-vector transfer, $Q$, the interference effects caused by the spatial correlation between neighbors become totally negligible and the so-called incoherent approximation applies [31]: the scattering process simply becomes a sum of singleatom collision events where the neutron transfers, by effect of the recoil, a large portion of its kinetic energy to the target atom. This energy, on average, is related to $Q$ by the simple relation: $\hbar \omega_{r}=(\hbar Q)^{2} / 2 M$, where $M$ is the mass of the recoiling particle [31]. In the experimental conditions where NCS applies, the momentum transfer (i.e. $\hbar Q$ ) becomes so large that the IA holds and the neutron scattering cross section is uniquely determined by the momentum distribution of the target atom [30].

The NCS experimental technique has widely been used, in recent times, with the aim of obtaining information on the momentum distribution function and the mean kinetic energy of dense quantum monatomic liquids [23,32-38]. However, when the same technique is extended to molecular liquids (e.g. $\mathrm{H}_{2}$ and $\mathrm{D}_{2}$ ) the situation appears more involved and the presence of an intra-molecular structure makes the interpretation of the experimental data much more difficult [39-44]. In practice, using NCS on the hydrogens, the energy and momentum transfers are so large that the scattering cross section is determined by the proton and deuteron (for hydrogen and deuterium, respectively) nuclear momentum distribution. In this condition, it becomes rather difficult (if not at all impossible) to obtain from the experimental data the corresponding function for the molecular COM.

A way of circumventing this difficulty was found by Langel et al. by using neutrons of lower energy [45]. Actually the intermolecular and intra-molecular degrees of freedom of the hydrogens, provided the system pressure is kept within reasonable limits, are decoupled to such an extent that they can be considered almost independent. In this situation, we can select an energy-interval of the probe such that the momentum transfer is large enough that the IA applies to the COM dynamics of the molecules but, at the same time, the energy transfer is low enough that the intra-molecular modes are not excessively excited. In this scenario it becomes possible to obtain the required information for the COM dynamics, provided a reliable model to describe the intra-molecular modes is available.

We have practically shown that this procedure is possible. In a recent series of experiments, the neutron spectrometer TOSCA, at the pulsed neutron source ISIS (Rutherford Appleton Laboratory, UK), was used to obtain a direct information on the translational mean kinetic energy (i.e. the COM's one) in liquid and solid parahydrogen $[46,47]$. In addition, we have shown that the lower energy portion (less than $50 \mathrm{meV}$ ) of the hydrogen spectrum derived from TOSCA can be efficiently used to gain information on the COM self-dynamics in the liquid phase $[48,49]$. In these experiments, a key role is played by the possibility of implementing the IA at the level of the molecular COM dynamics. Actually, this is rigorous only in the ideal limit: $Q \rightarrow \infty$, while some corrections should be applied for any finite value of $Q$. 


\section{The theoretical point of view}

As already mentioned in the previous section, in the case of low-pressure liquid $\mathrm{H}_{2}$ and $\mathrm{D}_{2}$ the decoupling between molecular COM dynamics and roto-vibrational dynamics holds almost exactly [50]. This fact implies that the double-differential neutron scattering cross-section, $\left(\mathrm{d} \sigma^{2} / \mathrm{d} \Omega \mathrm{d} E^{\prime}\right)$, is separable in the sum of two convolution products between COM and roto-vibrational parts, and, moreover, that the latter are substantially identical to the equivalent quantities observed in the gas phase [51,52]:

$$
\left(\frac{\mathrm{d} \sigma^{2}}{\mathrm{~d} \Omega \mathrm{d} E^{\prime}}\right)=\left(\frac{\mathrm{d} \sigma^{2}}{\mathrm{~d} \Omega \mathrm{d} E^{\prime}}\right)_{\mathrm{r}-\mathrm{v}} \otimes S_{\text {self, C }}(Q, \omega)+\left(\frac{\mathrm{d} \sigma^{2}}{\mathrm{~d} \Omega \mathrm{d} E^{\prime}}\right)_{\mathrm{r}-\mathrm{v}, \text { el. }} \otimes S_{\text {dist, C }}(Q, \omega)
$$

where $\left(\mathrm{d} \sigma^{2} / \mathrm{d} \Omega \mathrm{d} E^{\prime}\right)_{\mathrm{r}-\mathrm{v}}$ and $\left(\mathrm{d} \sigma^{2} / \mathrm{d} \Omega \mathrm{d} E^{\prime}\right)_{\mathrm{r}-\mathrm{v}, \text { el. }}$ are the roto-vibrational double-differential cross-sections (total and elastic, respectively) reported in detail in [51-53]. It is important to recall that these two functions are relatively simple (they can be even expressed analytically), since they are totally independent of the many-body quantum dynamics of liquid state. They are effected only by some isolated-molecule parameters, the $\mathrm{H}$ (or D) neutron scattering cross-section, and the composition of the rotationally-excited molecular population in the system. On the other hand, $S_{\text {self, C }}(Q, \omega)$ and $S_{\text {dist, C }}(Q, \omega)$ represent the well-known inelastic structure factors (self and distinct, respectively [31]), which describe the COM dynamics in the liquid state. It is worth noting that both of them exhibit the same properties (e.g. sum rules [54] etc.) as in a monatomic system, since the hydrogen intra-molecular structure has been confined within the other factors of the two convolution products.

In the limit of large transferred wave-vectors, where the COM static structure factor, $S_{\mathrm{C}}(Q)$ approaches the unity:

$$
S_{\mathrm{C}}(Q)=\int_{-\infty}^{\infty} \mathrm{d} \omega\left[S_{\text {self, } \mathrm{C}}(Q, \omega)+S_{\text {dist, } \mathrm{C}}(Q, \omega)\right] \simeq 1
$$

equation (9) reduces to:

$$
\left(\frac{\mathrm{d} \sigma^{2}}{\mathrm{~d} \Omega \mathrm{d} E^{\prime}}\right) \simeq\left(\frac{\mathrm{d} \sigma^{2}}{\mathrm{~d} \Omega \mathrm{d} E^{\prime}}\right)_{\mathrm{r}-\mathrm{v}} \otimes S_{\text {self, } \mathrm{C}}(Q, \omega)
$$

which is precisely the aforementioned incoherent approximation. Its physical meaning is clear and interesting: if $Q$ grows large enough, the collective excitations in a liquid system, described by $S_{\text {dist, C }}(Q, \omega)$, become more and more damped, to such an extent that, once $2 \pi / Q$ is much smaller than the typical intermolecular distance, they completely disappear. What is left is just the single-particle dynamics, which is actually contained in $S_{\text {self, C }}(Q, \omega)$. In liquid hydrogen [55] and deuterium [56], the incoherent limit appears as already reached at $Q=(5-6) \AA^{-1}$. Thus, in what follows in this section, we will always consider the response of liquid $\mathrm{H}_{2}$ and $\mathrm{D}_{2}$ at wave-vector transfers larger than this figure.

We have already pointed out that by further increasing the energy transfer the liquid responds like a collection of weakly interacting particles, as far as the mere 
COM dynamics is concerned. This is a general achievement, provided the intermolecular forces are not infinite anywhere, and implies that, in the asymptotic limit $(\omega, Q) \rightarrow \infty$, the self part of the COM structure factor, $S_{\text {self, C }}(Q, \omega)$, simply depends on the COM momentum distribution, $n_{\mathrm{C}}(P)$, through the well-known IA relationship:

$$
S_{\text {self, } \mathrm{C}}(Q, \omega)=\int \mathrm{d} \mathbf{P} n_{\mathrm{C}}(P) \delta\left(\omega-\frac{\hbar Q^{2}}{2 M}-\frac{\mathbf{P} \cdot \mathbf{Q}}{M}\right),
$$

where now $M$ represents the molecular mass. We will show in the rest of this section how to derive this formula from the short-time expansion of the intermediate scattering function and how to correct the IA for the so-called final states effects (FSE) at any finite value of $Q$.

Let us introduce the self part of the COM intermediate scattering function, $I_{\text {self, C }}(Q, t)$, as the Fourier transform of $S_{\text {self, C }}(Q, \omega)$ :

$$
I_{\text {self, } \mathrm{C}}(Q, t)=\int_{-\infty}^{\infty} \mathrm{d} \omega \exp (-\mathrm{i} \omega t) S_{\text {self, } \mathrm{C}}(Q, \omega)
$$

It is straightforward to prove [31] that the following relation holds:

$$
I_{\text {self, } \mathrm{C}}(Q, t)=\frac{1}{N}\left\langle\sum_{j=1}^{N} \exp \left(-\mathrm{i} \mathbf{Q} \cdot \mathbf{R}_{j}(t)\right) \exp \left(\mathrm{i} \mathbf{Q} \cdot \mathbf{R}_{j}(0)\right)\right\rangle
$$

where $N$ is the number of molecules in the system, $\mathbf{R}_{j}(t)$ is the position operator of the $j^{\text {th }} \mathrm{COM}$ at the time $t$, and the symbol $\langle\ldots\rangle$ represents the usual quantum statistical average. Using some standard algebra of the Heisenberg representation [57], one can rewrite the exponential operator at time $t, \exp \left(-\mathrm{i} \mathbf{Q} \cdot \mathbf{R}_{j}(t)\right)$, as:

$$
\exp \left(-\mathrm{i} \mathbf{Q} \cdot \mathbf{R}_{j}(t)\right)=\exp (\mathrm{i} H t) \exp \left(-\mathrm{i} H^{\prime} t\right) \exp \left(-\mathrm{i} \mathbf{Q} \cdot \mathbf{R}_{j}(0)\right),
$$

making use of the system Hamiltonian, $H$, and of the same operator modified by the scattering event concerning the $j^{\text {th }} \mathrm{COM}, H^{\prime}$ :

$$
H^{\prime}=H+\frac{\hbar^{2} Q^{2}}{2 M}+\frac{\hbar \mathbf{Q} \cdot \mathbf{P}_{j}}{M} .
$$

Inserting equations (15) and (16) into equation (14), and using again some Heisenberg-representation algebra [58], one finally obtains a complete expression for $I_{\text {self, C }}(Q, t)$ :

$$
I_{\text {self, } \mathrm{C}}(Q, t)=\exp \left(-\mathrm{i} \frac{\hbar Q^{2}}{2 M} t\right) \frac{1}{N}\left\langle\sum_{j=1}^{N} T_{t} \exp \left(-\mathrm{i} \int_{0}^{t} \mathrm{~d} \tau \frac{\mathbf{Q} \cdot \mathbf{P}_{j}(\tau)}{M}\right)\right\rangle
$$

where $T_{t}$ is the time-ordering operator, and $\mathbf{P}_{j}(\tau)=\exp (\mathrm{i} H \tau) \mathbf{P}_{j}(0) \exp (-\mathrm{i} H \tau)$ is the $\mathrm{COM}$ momentum at time $\tau$ in the Heisenberg representation. 
The IA is now obtained from the previous equation if the scattering time $t$ is assumed to be short, so that only times close to $t=0$ are retained. Practically it means that $\mathbf{P}_{j}(t)$ is expanded in a power series in $t: \mathbf{P}_{j}(t)=\mathbf{P}_{j}(0)+\mathbf{F}_{j}(0) t+\cdots$, where $\mathbf{F}_{j}$ is the force acting on the $j^{\text {th }} \mathrm{COM}$ due to the neighbor molecules, via the intermolecular potential. Keeping only the first term of the series, one writes:

$$
I_{\text {self, } \mathrm{C}}^{(\mathrm{IA})}(Q, t)=\exp \left(-\mathrm{i} \frac{\hbar Q^{2}}{2 M} t\right) \frac{1}{N}\left\langle\sum_{j=1}^{N} \exp \left(-\mathrm{i} \frac{\mathbf{Q} \cdot \mathbf{P}_{j}(0)}{M} t\right)\right\rangle
$$

whose Fourier transform yields:

$$
S_{\text {self, } \mathrm{C}}^{(\mathrm{IA})}(Q, \omega)=\frac{1}{N}\left\langle\sum_{j=1}^{N} \delta\left(\omega-\frac{\hbar Q^{2}}{2 M}-\frac{\mathbf{Q} \cdot \mathbf{P}_{j}(0)}{M}\right)\right\rangle,
$$

which is exactly identical to equation (12), since the only variable depending on the system is the momentum of the struck COM, $\mathbf{P}_{j}$.

We have obtained the IA just by neglecting the $\mathbf{F}_{j}(0)$ term in the expansion of $\mathbf{P}_{j}(t)$. So, for the IA to hold, one must have: $\hbar \mathbf{Q}+\mathbf{P}_{j} \gg \mathbf{F}_{j} \tau_{\mathrm{s}}$, where $\tau_{\mathrm{s}}$ is the typical scattering time. By increasing $Q, \tau_{\mathrm{s}}$ is of course reduced, but it is also crucial that $\mathbf{F}_{j}=-\partial V / \partial \mathbf{R}_{j}$ is not infinite anywhere (with $V$ being the intermolecular potential energy). In liquid hydrogens, $V$ has a steep repulsive, but not infinitely repulsive, core. Thus $\mathbf{F}_{j}$ is large, but still finite, and the IA can be attained. However, we expect that corrections to the IA could be relevant and it is important to determine the nature and the size of these deviations, following the procedure used in Ref. [59]. The expectation value in equation (17) may be expanded in cumulants, and, in addition, $\mathbf{P}_{j}(t)$ in each cumulant term may be expanded in a power series of $t$ :

$$
I_{\text {self, C }}(Q, t)=\exp \left(-\mathrm{i} \frac{\hbar Q^{2}}{2 M} t\right) \exp \left[\sum_{n=1}^{\infty} \frac{\mu_{n}(-\mathrm{i} t)^{n}}{n !}\right] \text {. }
$$

However, it is more useful to separate the IA and non-IA (i.e. FSE) contributions to the various cumulant coefficients. After having operated such a rearrangement, one obtains:

$$
I_{\text {self, } \mathrm{C}}(Q, t)=I_{\text {self, } \mathrm{C}}^{(\mathrm{IA})}(Q, t) \exp \left[\sum_{n=1}^{\infty} \frac{\beta_{n}(-\mathrm{i} t)^{n}}{n !}\right]
$$

where the first four $\beta_{n}$ coefficients can be evaluated making use of the quantum sum rules valid for isotropic $S_{\text {self, C }}(Q, \omega)[54]$ :

$$
\begin{aligned}
& \beta_{1}=0 \\
& \beta_{2}=0 \\
& \beta_{3}=\frac{\hbar Q^{2}}{6 M^{2} N}\left\langle\sum_{i=1}^{N} \nabla_{i}^{2} V\right\rangle ; \\
& \beta_{4}=\frac{Q^{2}}{3 M^{2} N}\left\langle\sum_{i=1}^{N}\left(\vec{\nabla}_{i} V\right)^{2}\right\rangle .
\end{aligned}
$$


Unfortunately a cumulant expansion cannot be terminated at the level of $\beta_{4}$, since $\beta_{4}>0$ and an unphysical divergence for $t \rightarrow \infty$ would occur in equation (21). For this reason another (coarser) approximation is usually applied, the so-called additive approach (AA) $[57,59]$. The AA is simply obtained by expanding the FSE part of equation (21) up to the $t^{5}$ term:

$$
I_{\text {self, } \mathrm{C}}^{(\mathrm{AA})}(Q, t) \simeq I_{\text {self, } \mathrm{C}}^{(\mathrm{IA})}(Q, t)\left(1+\frac{\mathrm{i} \beta_{3}}{3 !} t^{3}+\frac{\beta_{4}}{4 !} t^{4}-\frac{\mathrm{i} \beta_{5}}{5 !} t^{5}\right) .
$$

The convergence of $I_{\text {self, } \mathrm{C}}^{(\mathrm{AA})}(Q, t)$ for $t \rightarrow \infty$ is now fully guaranteed since $I_{\text {self, } \mathrm{C}}^{(\mathrm{IA})}(Q, t)$ exhibits at least a Gaussian dependence on $t$. By taking the Fourier transform of equation (23), one finally writes:

$$
S_{\text {self, } \mathrm{C}}^{(\mathrm{AA})}(Q, \omega) \simeq\left(1-\frac{\beta_{3}}{3 !} \frac{\partial^{3}}{\partial \omega^{3}}+\frac{\beta_{4}}{4 !} \frac{\partial^{4}}{\partial \omega^{4}}-\frac{\beta_{5}}{5 !} \frac{\partial^{5}}{\partial \omega^{5}}\right) S_{\text {self, } \mathrm{C}}^{(\mathrm{IA})}(Q, \omega) .
$$

which was first derived by Sears in 1969 [60]. Assuming a purely Gaussian COM momentum distribution (see section 1 for a discussion of this point), it is straightforward to write equation (24) analytically [59,61]:

$$
\begin{aligned}
S_{\text {self, } \mathrm{C}}^{(\mathrm{AA})}(Q, \omega) \simeq & \left\{1-\frac{\beta_{3}}{2 \mu_{2}^{2}}\left(\omega-\omega_{r}\right)\left[1-\omega_{d}^{2} / 3\right]+\frac{\beta_{4}}{8 \mu_{2}^{2}}\left[1-2 \omega_{d}^{2}+\omega_{d}^{4} / 3\right]\right. \\
& \left.+\frac{\beta_{5}}{8 \mu_{2}^{3}}\left(\omega-\omega_{r}\right)\left[1-2 \omega_{d}^{2} / 3+\omega_{d}^{4} / 15\right]\right\} S_{\text {self, } \mathrm{C}}^{(\mathrm{IA})}(Q, \omega),
\end{aligned}
$$

where $S_{\text {self, } \mathrm{C}}^{(\mathrm{IA})}(Q, \omega)$ retains a Gaussian functional form too:

$$
S_{\text {self, C }}^{(\mathrm{IA})}(Q, \omega)=\frac{1}{\sqrt{2 \pi \mu_{2}}} \exp \left[-\frac{\left(\omega-\omega_{r}\right)^{2}}{2 \mu_{2}}\right],
$$

with $\mu_{2}=\left\langle E_{k}\right\rangle 2 Q^{2} / 3 M, \omega_{r}=\hbar Q^{2} / 2 M$, and $\omega_{d}^{2}=\left(\omega-\omega_{r}\right)^{2} / \mu_{2}$. It is important to point out that now $\left\langle E_{k}\right\rangle$ represents the translational mean kinetic energy, i.e. the one of the molecular center-of-mass.

\section{Relevance of the FSE corrections in the TOSCA spectra}

\subsection{The TOSCA spectrometer}

The neutron scattering experiments were performed on the first version of the spectrometer (i.e. TOSCA-I), a crystal-analyzer inverse-geometry instrument operating at ISIS, the pulsed neutron source of Rutherford Appleton Laboratory (Chilton, Didcot, UK) [62]. The incident neutron beam spanned a broad energy $(E)$ range and the energy selection was carried out on the secondary neutron flightpath using the (002) Bragg reflection of 10 graphite single crystals placed in backscattering around $136.0^{\circ}$. This fixed the nominal scattered neutron energy, $E^{\prime}$, to 


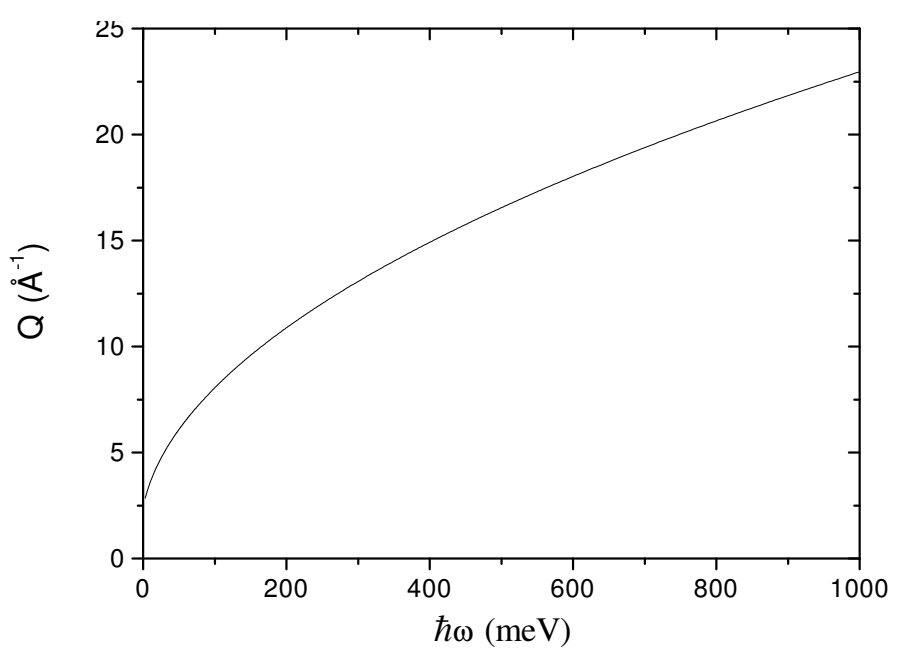

Figure 1. Average $(Q, \hbar \omega)$ trajectory in the kinematic plane spanned by the TOSCA spectrometer (back-scattering section).

$\simeq 3.51 \mathrm{meV}$. Higher order Bragg reflections were filtered out by 15 -cm thick beryllium blocks cooled down to $30 \mathrm{~K}$. This geometry allows to cover an extended energy transfer range, even though the fixed position of the crystal analyzers and the small value of the neutron final energy imply a variation in $Q$ which is a monotonic function of $\omega$. The resolving power of TOSCA-I was rather good $(2 \%<\Delta \hbar \omega / E<5 \%)$ in the energy transfer region relevant to the present experiment $(40 \mathrm{meV}<\hbar \omega<$ $1 \mathrm{eV}$ ). The extended spectral range of TOSCA makes this instrument a sort of neutron equivalent of a Raman optical spectrometer, the only difference being the momentum transfer monotonically growing along with the energy shift (see figure 1): $Q_{\omega \rightarrow \infty} \rightarrow\left(2 m_{n} \omega / \hbar\right)^{1 / 2}$, where $m_{n}$ is the neutron mass. Thus, intra-molecular transitions could be in principle easily observed even beyond the first vibrational transitions of molecular hydrogen and deuterium (we remind that these are placed at $515.6 \mathrm{meV}$ and at $370.9 \mathrm{meV}$, respectively [63]). However, it should be also pointed out that, due to the molecular recoil, the observed shifts are generally much greater than those observed on a conventional Raman spectrometer.

\subsection{The spectrum of liquid hydrogen}

In the high-energy region $(40 \mathrm{meV}<\hbar \omega<1 \mathrm{eV})$ of a TOSCA spectrum, the wave-vector transfer grows to such an extent $\left(5.6 \AA^{-1}<Q<23.0 \AA^{-1}\right)$ that the scattering process is expected to approach the IA regime, at least as far as liquid samples are concerned. Therefore, as we have already seen in section 2 , this spectrum would mainly contain information relative to the momentum distribution of a single particle in the system. On the other hand, in the TOSCA low-energy spectral region $(3 \mathrm{meV}<\hbar \omega<40 \mathrm{meV})$, the size of the wave-vector transfer $\left(2.9 \AA^{-1}<Q<5.6 \AA^{-1}\right)$ is of the order of the reciprocal of the inter-molecular distances between neighbors (namely, around $3.5 \AA$ for liquid $\mathrm{H}_{2}$ and $\mathrm{D}_{2}$ ). In this case, the self contribution to the scattering function is expected to provide information on 
the molecular dynamics driven by the pair interactions. Even though the transition between these two regimes is not clearly defined, it is reasonable to assume that in a liquid, it lies beyond the first peak of the static structure factor $S(Q)$ (which in liquid $\mathrm{H}_{2}$ and $\mathrm{D}_{2}$ is located around $Q=2 \AA^{-1}$ ), in the region where $S(Q)$ becomes $\simeq 1$ (i.e., as we have pointed out in section $2, Q \simeq 5-6 \AA^{-1}$ ). Should the momentum transfer decrease to much lower values, to such an extent that the hydrodynamic regime would drive the microscopic dynamics, it would be possible to gain direct information on the long-time behavior of the velocity auto-correlation function and the self-diffusion coefficient making use of the self part of the scattering function. But this is not exactly the case of TOSCA, and only an approximate estimate of the diffusive dynamics is expected to come out from the present hydrogen and deuterium spectra $[48,49]$.

Let us focus on liquid $\mathrm{H}_{2}$ : if pure liquid para-hydrogen is considered, the inelastic neutron spectrum becomes rather simple. At low temperature $(T<25 \mathrm{~K})$ only the fundamental rotational state $(J=0)$ is populated. In addition, since the transitions to the even states $\left(J=0 \rightarrow J^{\prime}=2,4,6, \cdots\right)$ are weighted by the small hydrogen coherent cross-section $\left(\sigma_{c}(H)=1.8\right.$ barn), their intensity is almost two orders of magnitude smaller than the transitions to the odd states (driven by the incoherent one, $\sigma_{i}(H)=80.3$ barn) [64]. Thus, the observed spectrum reduces, for any practical purpose, to a set of odd rotational transitions $\left(J=0 \rightarrow J^{\prime}=1,3,5, \cdots\right)$. Due to the small moment of inertia of hydrogen, rotational transitions are all well separated $\left(J=0 \rightarrow J^{\prime}=1\right.$ implies an energy jump of $14.7 \mathrm{meV}$, while $J=0 \rightarrow J^{\prime}=3$ corresponds to a jump of $87.4 \mathrm{meV}$ ). As a consequence, taking into account the extra shift induced by the molecular recoil, the overlap of different bands is small and each band can be analyzed almost individually. Applying equation (9) to lowtemperature para-hydrogen in the spectral region $(40 \mathrm{meV}<\hbar \omega<1 \mathrm{eV}$ ), the neutron scattering double-differential cross-section simply becomes:

$$
\left(\frac{\mathrm{d}^{2} \sigma}{\mathrm{d} \Omega \mathrm{d} E^{\prime}}\right)=\frac{k^{\prime}}{k} \frac{\sigma_{i}(H)}{4 \pi} \sum_{J^{\prime}=1,3,5, \cdots}|f(Q)|_{0 \rightarrow J^{\prime}}^{2} S_{\text {self, } \mathrm{C}}(Q, \omega) \otimes \delta\left(\omega-\omega_{0 \rightarrow J^{\prime}}\right),
$$

where $k$ and $k^{\prime}$ represent the incoming and out-coming neutron wave-vector, respectively; $f(Q)$ is the intra-molecular form factor [53], and $\hbar \omega_{0 \rightarrow J^{\prime}}$ stands for the energy gap between the $J=0$ and the various $J^{\prime}$ rotational states. Actually a portion of the high-energy region of the spectra (namely, $100 \mathrm{meV}<\hbar \omega<1 \mathrm{eV}$ ), which involves the rotational transitions beyond the first excited rotational level, $\left(J=0 \rightarrow J^{\prime}=3,5,7\right)$ has been already analyzed in the framework of the pure IA $[46,47]$. In this case, due to the high value of the wave-vector $\left(Q>8.0 \AA^{-1}\right)$ and energy transfers, the inter-molecular interactions were supposed only to give rise to a simple renormalization of the COM mean kinetic energy. This, in turn, affects the width of the molecular recoil peak which describes the free motion of the molecule. A simple modification of the Young-Koppel theory [53], originally devised for gaseous $\mathrm{H}_{2}$ and $\mathrm{D}_{2}$, was applied and we could determine the COM mean kinetic energy in liquid hydrogen and deuterium as a function of the thermodynamic conditions $[46,47]$. As we see at the end of this subsection, a careful investigation of 
the TOSCA spectra will show important deviations from IA, even in this high- $\omega$ scattering region.

The measurement was carried out in the dense liquid phase at $T=19.34 \mathrm{~K}$. After performing the background measurements of the empty cryostat, we cooled the empty container at the desired temperature and we measured its time-of-flight (TOF) neutron spectrum. Then, hydrogen was allowed to condense in the scattering cell. This was made of SS-316 steel and consisted of an array of 5 tubes (O.D. $3.2 \mathrm{~mm}$, I.D. $1.6 \mathrm{~mm}$ ), $64.0 \mathrm{~mm}$ high and $5.5 \mathrm{~mm}$ apart from one another, so as to minimize the multiple scattering contributions. After filling up the cell, the pressure of the gas handling system was set to $p=191$ bar (much larger than the corresponding saturated vapor pressure: $p_{\mathrm{SVP}} \simeq 0.8$ bar [65]) in order to obtain a high-density liquid sample: $n=25.43 \mathrm{~nm}^{-3}$ [65]. The reason for this requirement will be made clear hereinafter. At the bottom of the scattering container, out of the neutron beam, we had inserted some powder of a paramagnetic catalyst made of $\mathrm{Cr}_{2} \mathrm{O}_{3}$ on an $\mathrm{Al}_{2} \mathrm{O}_{3}$ substrate in order to speed up the conversion from ortho- to para-hydrogen. The relative concentration of the two species was monitored observing the scattering spectrum. In particular, we could observe the progressive disappearance of the $J=$ $1 \rightarrow J^{\prime}=1$ (quasi-elastic line) transition, which is weighted by the incoherent crosssection of the proton [31], from the low energy portion of the spectrum. When this spectral feature was below the limit of detectability (in practice, masked by the $J=$ $0 \rightarrow J^{\prime}=0$ transition, which is weighted by the coherent cross-section of the proton) we assumed that the equilibrium had been reached. The equilibration process lasted, in our case, about 20-25 hours. The estimated concentration of para-hydrogen, based on the theoretical calculation [65], was assumed to be $99.82 \%$. Then, we started recording the scattering spectrum up to an integrated proton current of $3065.8 \mu \mathrm{A} \mathrm{h}$ (roughly, 18 hours of beam time). The stability of the thermodynamic conditions during the experiment was quite good: temperature fluctuations never exceeded $0.2 \mathrm{~K}$ and the pressure stability (2 bar) was strictly related to this value due to the good realization of the gas handling system. The temperature uncertainty (standard deviation) estimated for our measurement was $0.02 \mathrm{~K}$, and the density of our sample (and its uncertainty) was later derived according to [65]: $n=25.43(3) \mathrm{nm}^{-3}$. In figure 2(a) we show the raw spectrum of liquid para-hydrogen at $T=19.34 \mathrm{~K}$ and $n=25.43 \mathrm{~nm}^{-3}$ in all the TOSCA-accessible $\omega$-range.

The experimental TOF spectra were transformed into energy transfer data, detector by detector, making use of the standard TOSCA-I routines available on the spectrometer, and then added together exploiting the narrow angular range spanned by the whole set of detectors $\left(\Delta \theta=9.5^{\circ}\right)$ [62]. In this way, we produced a single double-differential cross-section measurement along the TOSCA-I kinematic path $(Q, \omega)$. Then, data were corrected for the $k^{\prime} / k$ factor and subtracted of the tiny empty can contribution (separately recorded at $T=34.99(5) \mathrm{K}$, with an integrated proton current of $982.2 \mu \mathrm{Ah}$ ). Two fundamental corrections were carefully performed: multiple scattering evaluation and self-absorption attenuation. As far as the former is concerned, we simulated both single-scattering and multiple-scattering neutron spectra measured by TOSCA-I for each sample through the analytical ap- 

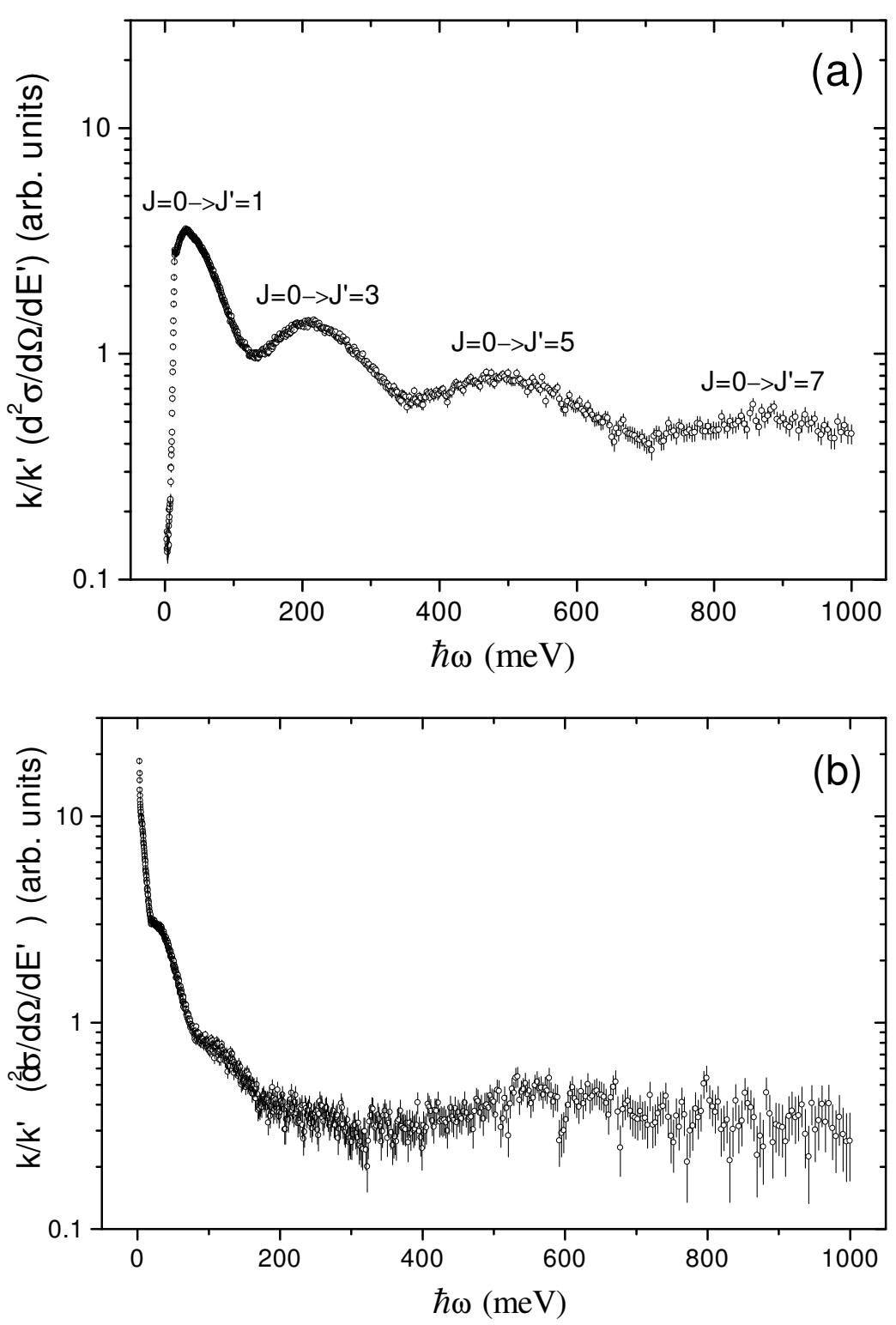

Figure 2. (a) Raw experimental neutron spectrum measured on liquid parahydrogen at $T=19.34 \mathrm{~K}$ (after the can scattering subtraction). The rotational transitions from the $J=0$ ground state to the odd- $J^{\prime}$ excited states are clearly identified. (b) Raw experimental neutron spectrum measured on liquid orthodeuterium at $T=21.3 \mathrm{~K}$ (after the can scattering subtraction). 
proach suggested by Agrawal in the case of an infinite flat slab-like sample [66]. All the details of this procedure have been reported in [49]. Following this stage, selfabsorption correction was applied to the normalized single-scattering experimental data, still assuming the infinite slab approximation and making use of the aforementioned analytical approach [66]. However, in this case, no model was employed for the para-hydrogen total scattering cross-section, which, on the contrary, was obtained from the experimental results of direct measurements on the SVP liquid $\mathrm{p}-\mathrm{H}_{2}$ at $T=16.0(2) \mathrm{K}[67]$. At the end of the correction procedure, the processed data, limited to the $3 \mathrm{meV}<\hbar \omega<1 \mathrm{eV}$ interval, had actually become proportional to the double-differential neutron scattering cross-section, as in equation (11), plus a small (and rather flat) sample-dependent background. Making use of the aforementioned Young-Koppel model [52,53], we were able to calculate the intra-molecular term of equation $(11),\left(\mathrm{d} \sigma^{2} / \mathrm{d} \Omega \mathrm{d} E^{\prime}\right)_{\mathrm{r}-\mathrm{v}}$, along the $(Q, \hbar \omega)$ trajectory in the kinematic plane spanned by TOSCA-I. Assuming, as a first working hypothesis, a simple IA form for $S_{\text {self, C }}(Q, \omega)$ in the $40 \mathrm{meV}<\hbar \omega<1 \mathrm{eV}$ range, we set up a simple fitting procedure of the processed experimental data, $\Sigma_{\exp }(Q, \omega)$, through the following function:

$$
\Sigma_{\exp }(Q, \omega)=\left[A \frac{k}{k^{\prime}}\left(\frac{\mathrm{d} \sigma^{2}}{\mathrm{~d} \Omega \mathrm{d} E^{\prime}}\right)_{\mathrm{r}-\mathrm{v}} \otimes S_{\mathrm{self}, \mathrm{C}}^{(\mathrm{IA})}(Q, \omega)+B_{\mathrm{II}}(\omega)\right] \otimes R_{\mathrm{Tosca}}(\omega)
$$

where $A$ represents an overall normalization constant, $B_{\mathrm{II}}(\omega)$ accounts for a polynomial (second order) sample-dependent background, and $R_{\text {Tosca }}(\omega)$ stands for the instrumental energy resolution [62]. We recall here that this fitting function contained only five independent parameters: $A$, three polynomial coefficients in $B_{\mathrm{II}}(\omega)$, and $\left\langle E_{k}\right\rangle$, which is implied by $S_{\text {self, }}^{(\mathrm{IA})}(Q, \omega)$ as shown in equation $(26)$. The fit, performed through a FORTRAN code coupled with the MINUIT minimization library [68], showed that equation (28) does not properly describe the liquid $\mathrm{p}-\mathrm{H}_{2}$ scattering law in the $40 \mathrm{meV}<\hbar \omega<1 \mathrm{eV}$ range, since the reduced $\chi^{2}$ could not decrease below 6.83 (see also figure 3(a)). Thus, in order to improve the quality of our fit, we replaced $S_{\text {self, }}^{(\mathrm{IA})}(Q, \omega)$ with $S_{\text {self, C }}^{(\mathrm{AA})}(Q, \omega)$ in equation $(28)$, the latter function being defined as in equation (25). As far as the AA coefficients are concerned, $\beta_{3}$ and $\beta_{4}$ were evaluated following equation (22) and assuming a pair-wise additive inter-molecular potential:

$$
\begin{aligned}
\frac{1}{N}\left\langle\sum_{j=1}^{N} \nabla_{j}^{2} V\right\rangle & =n \int \mathrm{d} \mathbf{R} g(R) \nabla^{2} v(R)=169.8 \mathrm{meV}^{-2}, \\
\frac{1}{N}\left\langle\sum_{j=1}^{N}\left(\vec{\nabla}_{j} V\right)^{2}\right\rangle & =n \int \mathrm{d} \mathbf{R} g(R)(\vec{\nabla} v(R))^{2}=1800 \mathrm{meV}^{2} \AA^{-2},
\end{aligned}
$$

where $n$ is the molecular density of the system, $v(R)$ the Silvera-Goldman pair-wise inter-molecular potential [69], and $g(R)$ is the COM pair correlation function, derived from previous PIMC simulations [47]. On the contrary, $\beta_{5}$ was supposed to have only a $Q^{2}$ dependence (in the framework of the well-known Gaussian approximation for $\left.I_{\text {self, C }}(Q, t)[54]\right)$, and was left as an additional fitting parameter (the sixth). After 
repeating the fitting procedure including the FSE terms of the AA, we obtained a much better description of the experimental data, since reduced $\chi^{2}$ stabilized this time around 1.48. The improvement of the fitting quality induced by the FSE can be clearly observed in figure 3(a). The experimental estimate of the COM mean kinetic energy was $\left\langle E_{k}\right\rangle=78(4) \mathrm{K}$.

In order to verify that the insufficiency of the IA to properly describe the experimental data from liquid para-hydrogen was caused by the inter-molecular FSE, we decided to study a low-density gaseous sample $\left(T=28.94(2) \mathrm{K}, n=2.06(9) \mathrm{nm}^{-3}\right.$, $\left.c\left[\mathrm{p}-\mathrm{H}_{2}\right]=97.7(2) \%\right)$. As a matter of fact, by increasing the mean free path by a factor of 2.3, we expected a much lower effect of the FSE on the measured neutron spectrum. The gaseous sample $(3.5-\mathrm{mm}$ thick at $p=6.0(2)$ bar) was measured on TOSCA-I until an integrated proton current of $745 \mu \mathrm{A} \mathrm{h}$ was reached. Then, its spectrum was processed similar to the aforementioned liquid $\mathrm{p}-\mathrm{H}_{2}$ data set. A purely IA fitting procedure was performed in a wide energy-transfer range: $4 \mathrm{meV}<\hbar \omega<1 \mathrm{eV}$. The final results can be seen in figure $3(\mathrm{c})$, where a very good agreement between $\Sigma_{\exp }(Q, \omega)$ and the IA model is evident, the reduced $\chi^{2}$ turning out to be 1.35. The experimental translational mean kinetic energy, in this case, was estimated to be: $\left\langle E_{k}\right\rangle=44(3) \mathrm{K}$, which is compatible with the classical value: $\left\langle E_{k}\right\rangle_{\mathrm{cl}}=43.41(3) \mathrm{K}$. It is worth noting that, as far as gaseous para-hydrogen is concerned, our findings totally confirm the conclusions drawn by Simmons et al. [70] about the validity of the Young-Koppel model.

\subsection{The spectrum of liquid deuterium}

Let us now describe the liquid- $\mathrm{D}_{2}$ inelastic scattering: even though pure liquid ortho-deuterium is considered, its neutron spectrum is not as simple as in the para-hydrogen case. We still have that at low temperature $(T<25 \mathrm{~K})$ only the fundamental rotational state $(J=0)$ is populated; but in o- $\mathrm{D}_{2}$ the transitions to the even states $\left(J=0 \rightarrow J^{\prime}=0,2,4,6, \cdots\right)$ are weighted by a linear combination of the coherent and incoherent atomic cross-sections [64]: $\sigma_{c}(D)+5 \sigma_{i}(D) / 8=6.87$ barn. Thus, their intensity is larger (but just by one order of magnitude) than the transitions to the odd states (driven by a fraction of incoherent atomic cross-sections [64]: $3 \sigma_{i}(D) / 8=0.77$ barn), which are, actually, small but not totally negligible. In addition, the spectral framework is complicated by the moment of inertia of deuterium, so that the rotational transitions are not well separated as in $\mathrm{p}-\mathrm{H}_{2}\left(J=0 \rightarrow J^{\prime}=0\right.$ implies no energy jump, while $J=0 \rightarrow J^{\prime}=2$ corresponds to a jump of $22.2 \mathrm{meV}$ only). This situation is clearly seen in figure $2(\mathrm{~b})$, where the individual transition peaks are hardly recognizable. Finally, a further complication is caused by the socalled elastic transition (i.e. $J=0 \rightarrow J^{\prime}=0$ ), which, according to equation (9), also implies an additional term related to the collective excitations of the liquid and expressed by $S_{\text {dist, C }}(Q, \omega)$. However, limiting our data analysis to the $50 \mathrm{meV}<\hbar \omega<1$ eV range, we make sure that $S_{\text {dist, }}(Q, \omega)$ could be totally neglected, as explained in section 2. Summarizing the discussion above, we conclude that equation (9) applied to low-temperature ortho-deuterium in the spectral region $50 \mathrm{meV}<\hbar \omega<1 \mathrm{eV}$ 

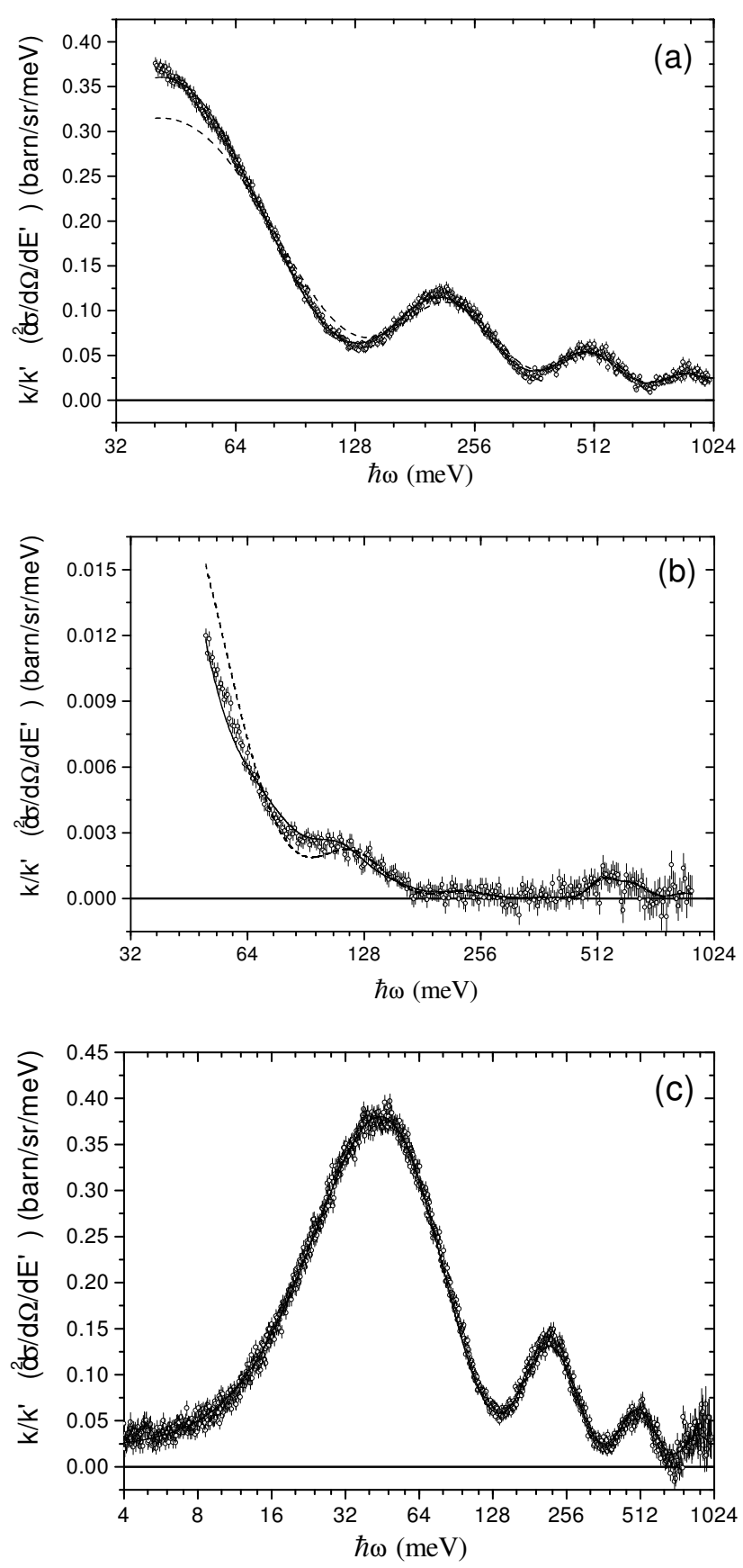

Figure 3. (a) Experimental double-differential neutron scattering cross-section from liquid para-hydrogen at $T=19.34 \mathrm{~K}$ (circles with error bars) together with its impulse-approximation (dashed line) and additive-approach (full line) best fits. (b) Experimental double-differential neutron scattering cross-section from liquid ortho-deuterium at $T=21.3 \mathrm{~K}$ (circles with error bars) together with its impulse-approximation (dashed line) and additive-approach (full line) best fits. (c) Experimental double-differential neutron scattering cross-section from gaseous para-hydrogen at $T=28.94 \mathrm{~K}$ (circles with error bars) together with its impulse-approximation (full line) best fit. 
reads as:

$$
\left(\frac{\mathrm{d}^{2} \sigma}{\mathrm{d} \Omega \mathrm{d} E^{\prime}}\right)=\frac{k^{\prime}}{k} \sum_{J^{\prime}=0,1,2,3, \cdots} \frac{\sigma_{J^{\prime}}}{4 \pi}|f(Q)|_{0 \rightarrow J^{\prime}}^{2} S_{\text {self, } \mathrm{C}}(Q, \omega) \otimes \delta\left(\omega-\omega_{0 \rightarrow J^{\prime}}\right),
$$

where $\sigma_{J^{\prime}}=6.87$ barn if $J^{\prime}$ is even, and $\sigma_{J^{\prime}}=0.77$ barn if $J^{\prime}$ is odd.

The ortho-deuterium measurement was carried out in the liquid phase at $T=$ 21.3 K using a flat scattering cell. This was made of aluminum, $1.2 \mathrm{~mm}$ thick walls, with a cylindrical-slab geometry. The sample thickness was $2.5 \mathrm{~mm}$ and the cell diameter $(55.0 \mathrm{~mm})$ was a little larger than the beam cross-section (roughly 50 $\mathrm{mm} \times 20 \mathrm{~mm}$ ). After filling up the cell, the pressure of the gas handling line was set to $p=0.76$ bar (slightly larger than the corresponding saturated vapor pressure: $p_{\mathrm{SVP}} \simeq 0.47$ bar [71]) in order to make sure that the scattering cell was actually totally filled with liquid. As in the case of para-hydrogen, at the bottom of the sample container (out of the neutron beam) we had placed some paramagnetic catalyst to accelerate the conversion from para- to ortho-deuterium. The relative concentration of the two deuterium species was checked observing the scattering spectrum in the spectral region $3 \mathrm{meV}<\hbar \omega<200 \mathrm{meV}$. When the change in the aforementioned $\hbar \omega$ range was below the statistical limit of detectability, we assumed that the equilibrium had been reached. This process took about 23 hours and the estimated final concentration [71] of o- $\mathrm{D}_{2}$ in the sample was $97.4 \%$. After the end of the equilibration, we started measuring the scattering spectrum up to an integrated proton current of $2062.5 \mu \mathrm{A} \mathrm{h}$ (equivalent to roughly 12 hours of beam time). The temperature and pressure uncertainties (standard deviations) were estimated to be $0.3 \mathrm{~K}$ and 0.02 bar, respectively. The density of our sample (and its uncertainty) was later derived according to [71]: $n=25.2(1) \mathrm{nm}^{-3}$. In figure $2(\mathrm{~b})$ we show the raw spectrum of liquid ortho-deuterium at $T=21.3 \mathrm{~K}$ and $n=25.2 \mathrm{~nm}^{-3}$ in all the TOSCA-accessible $\omega$-range.

Experimental TOF data from the liquid o- $\mathrm{D}_{2}$ were processed following a similar, but somewhat simpler guide-line like the one already employed for $\mathrm{p}-\mathrm{H}_{2}$ (see the previous subsection for details): the multiple-scattering contribution was estimated to be roughly $6.1 \%$ of the single-scattering one [72], and then it was neglected and included in the background (see below). As far as the self-shielding is concerned, since liquid ortho-deuterium exhibits an almost constant total scattering cross-section [73] in the range of interest of the incoming neutron energy (53 meV $<E<303 \mathrm{meV}$, see later), we decided not to perform any corrections. Once obtained the experimental $\Sigma_{\exp }(Q, \omega)$, we applied to the processed data a IA fitting procedure similar to the one expressed in equation (28), just replacing the parabolic polynomial sample-dependent background by a more general one of the third order. The fit was accomplished in the energy transfer range $50 \mathrm{meV}<\hbar \omega<1 \mathrm{eV}$, even though the main data features extended up to about $\hbar \omega=300 \mathrm{meV}$. The results, reported in figure $3(\mathrm{~b})$, were not totally satisfactory since the reduced $\chi^{2}$ did not decrease below 2.70. Following the same strategy as for liquid para-hydrogen, the leading terms of the FSE were introduced in the fitting procedure through the AA, just replacing $S_{\text {self, C }}^{(\mathrm{IA})}(Q, \omega)$ with $S_{\text {self, C }}^{(\mathrm{AA})}(Q, \omega)$. Its FSE coefficients were either 
estimated $\left(\beta_{3}\right.$ and $\left.\beta_{4}\right)$, or left as a fitting parameter $\left(\beta_{5}\right)$. The former two were calculated following equations (22) and (29) using a PIMC pair correlation function (see [74] for the details of the simulation technique), and the potential derivative terms turned out to be:

$$
\begin{aligned}
\frac{1}{N}\left\langle\sum_{i=1}^{N} \nabla_{i}^{2} V\right\rangle & =145.5 \mathrm{meV}^{-2} \\
\frac{1}{N}\left\langle\sum_{i=1}^{N}\left(\vec{\nabla}_{i} V\right)^{2}\right\rangle & =981.1 \mathrm{meV}^{2} \AA^{-2} .
\end{aligned}
$$

The new fitting procedure achieved a much better agreement between experimental data and model function (reduced $\chi^{2}=1.08$ ), as seen in figure $3(\mathrm{~b})$, and the COM mean kinetic energy was evaluated to be $\left\langle E_{k}\right\rangle=65(3) \mathrm{K}$.

\section{Discussion and conclusions}

The result for the center-of-mass mean kinetic energy of liquid para-hydrogen (namely $\left\langle E_{k}\right\rangle=78(4) \mathrm{K}$ ) was found in good agreement with the corresponding Path Integral Monte Carlo simulation value: $\left\langle E_{k}\right\rangle^{(\mathrm{PIMC})}=74.5(1) \mathrm{K}$ [47]. The same quality of agreement is observed if the present experimental result is compared to the $\left\langle E_{k}\right\rangle$ value obtained from the same experimental data through a simpler IA analysis in a narrower energy transfer range $(100 \mathrm{meV}<\hbar \omega<1 \mathrm{eV}):\left\langle E_{k}\right\rangle=74(5) \mathrm{K}$ [47]. Unfortunately, as for liquid ortho-deuterium, it is not possible to establish such a comparison: the most relevant spectral features lie in the $50 \mathrm{meV}<\hbar \omega<100 \mathrm{meV}$ range, where, on the other hand, the FSE are not negligible. However, making use of other neutron spectrometers exploring different $(Q, \omega)$ trajectories, it was feasible to extract information on the translational mean kinetic energy from a liquid normal (i.e. $66 . \overline{6} \%$ of ortho) $\mathrm{D}_{2}$ sample at $T=20.0 \mathrm{~K}$ and $n=25.7 \mathrm{~nm}^{-3}$. After a pure IA fit over an extended energy and momentum transfer range $(15 \mathrm{meV}<\hbar \omega<120 \mathrm{meV}$ and $\left.6 \AA^{-1}<Q<15 \AA^{-1}\right)$, Mompeán et al. [75] managed to estimate the COM mean kinetic energy to be $\left\langle E_{k}\right\rangle=60(9) \mathrm{K}$. This figure can be easily reconciled with our present finding: $\left\langle E_{k}\right\rangle=65(3) \mathrm{K}$, confirming that there is no appreciable effect of the roto-vibrational molecular population on the COM dynamics in liquid deuterium.

As we mentioned in section 4 , in the case of liquid para-hydrogen, where there are virtually no difficulties caused by the elastic $\left(J=0 \rightarrow J^{\prime}=0\right)$ scattering, a careful data analysis in the low-energy transfer range $3 \mathrm{meV}<\hbar \omega<40 \mathrm{meV}$ has been attempted, and successfully carried out [49], making use of the Gaussian approximation in conjunction with a model for power spectrum of the velocity autocorrelation function of the COM, $f(\omega)$ [54]. It is worth noting that the link between the present AA description of the FSE and the velocity auto-correlation function approach exists and can be shown in a straightforward way. According to the Gaussian 
approximation [54], $I_{\text {self, } \mathrm{C}}(Q, t)$ generally writes as:

$$
I_{\text {self, } \mathrm{C}}(Q, t)=\exp \left(-\mathrm{i} \frac{\hbar Q^{2}}{2 M} t\right) \exp \left[-Q^{2} w(t)\right]
$$

where $w(t)$ is the mean squared travelled path of the COM at time $t$, and is simply related to $f(\omega)$ by $[49,54]$ :

$$
w(t)=\frac{\hbar}{2 M} \int_{0}^{\infty} \mathrm{d} \omega \frac{f(\omega)}{\omega}\left\{[1-\cos (\omega t)] \operatorname{coth}\left(\hbar \omega / 2 k_{\mathrm{B}} T\right)+\mathrm{i}[\sin (\omega t)-\omega t]\right\} .
$$

Since, as we have seen in section 3, we are interested only in the short-time behavior of $I_{\text {self, }}(Q, t)$, let us expand $w(t)$ in a power series in $t$ :

$$
w(t) \simeq w(0)+w(0)^{(1)} t+\frac{w(0)^{(2)}}{2 !} t^{2}+\frac{w(0)^{(3)}}{3 !} t^{3}+\frac{w(0)^{(4)}}{4 !} t^{4} .
$$

Comparing equation (20) and equation (34), and then plugging the latter into equation (33), one obtains:

$$
\begin{aligned}
& Q^{2} w(0)=0 ; \\
& Q^{2} w(0)^{(1)}=\mathrm{i} \mu_{1}=0 ; \\
& Q^{2} w(0)^{(2)}=\mu_{2}=\frac{\hbar Q^{2}}{2 M} \int_{0}^{\infty} \mathrm{d} \omega f(\omega) \omega \operatorname{coth}\left(\hbar \omega / 2 k_{\mathrm{B}} T\right) ; \\
& Q^{2} w(0)^{(3)}=-\mathrm{i} \mu_{3}=-\mathrm{i} \beta_{3}=\frac{-\mathrm{i} \hbar Q^{2}}{2 M} \int_{0}^{\infty} \mathrm{d} \omega f(\omega) \omega^{2} ; \\
& Q^{2} w(0)^{(4)}=-\mu_{4}=-\beta_{4}=\frac{-\hbar Q^{2}}{2 M} \int_{0}^{\infty} \mathrm{d} \omega f(\omega) \omega^{3} \operatorname{coth}\left(\hbar \omega / 2 k_{\mathrm{B}} T\right) .
\end{aligned}
$$

Making use of the $f(\omega)$ sum-rules reported in [76], it is possible to verify that the previous relationships on the one hand, and the formula $\mu_{2}=2 Q^{2} / 3 M\left\langle E_{k}\right\rangle$ together with those in equation (22), on the other, coincide, provided the COM momentum distribution has a zero kurtosis [57], as in a purely Gaussian functional form.

In conclusion, in the present study we have measured, making use of the TOSCA crystal-analyzer inverse-geometry spectrometer, the neutron scattering spectrum of liquid para-hydrogen (at $T=19.3 \mathrm{~K}$ and $n=25.4 \mathrm{~nm}^{-3}$ ) and ortho-deuterium (at $T=21.3 \mathrm{~K}$ and $n=25.2 \mathrm{~nm}^{-3}$ ) in the energy and momentum transfer ranges $3 \mathrm{meV}<\hbar \omega<1 \mathrm{eV}$ and $2.9 \AA^{-1}<Q<23.0 \AA^{-1}$, respectively. From the highenergy and high-momentum region of the spectrum (namely $40 \mathrm{meV}<\hbar \omega<1 \mathrm{eV}$, and $5.6 \AA^{-1}<Q<23.0 \AA^{-1}$ ), where the incoherent approximation for the centerof-mass scattering law surely holds, we have been able to extract the COM (i.e. translational) mean kinetic energy through a fit of the experimental spectra by applying the well-known Young-Koppel model to describe the intra-molecular rotovibrational dynamics of $\mathrm{H}_{2}$ and $\mathrm{D}_{2}$. As expected, the mean kinetic energy turned out to be greatly larger than the corresponding classical value (i.e. $1.5 k_{\mathrm{B}} T$ ), owing to the 
presence of relevant quantum effects in both liquids. However, given the almost identical thermodynamic conditions, liquid para-hydrogen appeared rather more quantal than ortho-deuterium: $\left\langle E_{k}\right\rangle=78(4) \mathrm{K}$ (para-hydrogen), and $\left\langle E_{k}\right\rangle=65(3) \mathrm{K}$ (orthodeuterium). Moreover, significant deviations from the impulse approximation have been detected during the fitting procedure of both sample data (especially in the 40-50 meV $<\hbar \omega<100 \mathrm{meV}$ interval). Thus, the inclusion of some correction terms (accounting for the so-called final state effects following Glyde's additive approach) was necessary in order to accurately describe the experimental spectra. We also proved that this approach is a simple time-expansion of the well-known Gaussian approximation, provided the COM momentum distribution exhibits a purely Gaussian functional form. The first two coefficients controlling the intensity of the final state effects were evaluated making use of the Silvera-Goldman inter-molecular potential and the center-of-mass pair correlation functions of liquid $\mathrm{H}_{2}$ and $\mathrm{D}_{2}$. The comparison of the present translational mean kinetic energy values with the available experimental (on o- $\mathrm{D}_{2}$ ) and simulated data (on $\mathrm{p}-\mathrm{H}_{2}$ ) in the literature is quite satisfactory and confirms the excellent performances of TOSCA in the spectroscopic analysis of the condensed phases of liquid hydrogens.

\section{Acknowledgements}

This work has been financially supported by C.N.R. (Italy). The authors acknowledge the skillful technical support of the ISIS Sample Environment Section. One of the authors (G.C.) is also indebted to Accademia Nazionale dei Lincei (Italy) for the research grant Borsa di Perfezionamento negli Studi di Spettroscopia Neutronica "F.P.Ricci".

\section{References}

1. Barocchi F., Neumann M., Zoppi M. // Phys. Rev. A, 1985, vol. 31, p. 4015; Barocchi F., Neumann M., Zoppi M. // Phys. Rev. A, 1987, vol. 36, p. 2440.

2. Marinin V.S., Pashkov V.V. // Sov. J. Low Temp. Phys., 1977, vol. 3, p. 397; Marinin V.S., Pashkov V.V. // Sov. J. Low Temp. Phys., 1977, vol. 3, p. 472.

3. Dyugaev A.M. // J. Low Temp. Phys., 1990, vol. 78, p. 79.

4. Ceperley D.M. // Phys. Rev. Lett., 1992, vol. 69, p. 331.

5. Ceperley D.M. // Rev. Mod. Phys., 1995, vol. 67, p. 279.

6. Voth G., Chandler D., Miller V.H. // J. Chem. Phys., 1989, vol. 91, p. 7749.

7. Cao J., Voth G. // J. Chem. Phys., 1993, vol. 99, p. 10070.

8. Cao J., Voth G. // J. Chem. Phys., 1994, vol. 100, p. 5093; 1994, vol. 100, p. 5106; 1994, vol. 101, p. 6157 ; 1994, vol. 101, p. 6168.

9. Martyna G.J. // J. Chem. Phys., 1996, vol. 104, p. 2018.

10. Cao J., Martyna G.J. // J. Chem. Phys., 1996, vol. 104, p. 2028.

11. Pavese M., Voth G. // Chem. Phys. Lett., 1996, vol. 249, p. 231; Calhoun A., Pavese M., Voth G. // ibid., 1996, vol. 262, p. 415.

12. Kinugawa K. // Chem. Phys. Lett., 1998, vol. 292, p. 454. 
13. Balucani U., Zoppi M. Dynamics of the Liquid State. Oxford, Oxford University Press, 1994.

14. Hansen J.P., Mc Donald I. Theory of Simple Liquids. London, Academic Press, 1986.

15. Andreev A.F. // JETP Lett., 1978, vol. 28, p. 556; Andreev A.F., Kosevich Yu.A. // Sov. Phys. JETP, 1979, vol. 50, p. 1218.

16. Feenberg E. Theory of Quantum Fluids. New York and London, Academic Press, 1969.

17. Ceperley D.M., Pollock E.L. // Phys. Rev. Lett., 1986, vol. 56, p. 351; Can J. // Phys., 1987, vol. 65, p. 1416.

18. Sosnick T.R., Snow W.M., Sokol P.E. // Phys. Rev. B, 1990, vol. 41, p. 11185.

19. Azuah R.T., Stirling W.G., Glyde H.R., Sokol P.E., Bennington S.M. // Phys. Rev. B, 1995, vol. 51, p. 605.

20. Azuah R.T., Stirling W.G., Glyde H.R., Sokol P.E., Bennington S.M., Boninsegni M. // Phys. Rev. B, 1997, vol. 56, p. 14620.

21. Andersen K.H., Stirling W.G., Glyde H.R. // Phys. Rev. B, 1997, vol. 56, p. 8978.

22. Glyde H.R., Azuah R.T., Stirling W.G. // Phys. Rev. B, 2000, vol. 62, p. 14337.

23. Timms D.N., Evans A.C., Boninsegni M., Ceperley D. M., Mayers J., Simmons R.O. // J. Phys.: Condens. Matter, 1996, vol. 8, p. 6665; Azuah R.T., Stirling W.G., Glyde H.R., Boninsegni M. // J. Low Temp. Phys., 1997, vol. 109, p. 287.

24. Neumann M., Zoppi M. // Phys. Rev. E, 2002, vol. 65, p. 31203.

25. Landau L.D., Lifshits E.M. Statistical Physics. Vol. I. Moskow, Nauka, 1978.

26. Woods A.D.B., Sears V.F. // Phys. Rev. Lett., 1977, vol. 39, p. 415.

27. Celli M., Zoppi M., Mayers J. // Phys. Rev. B, 1998, vol. 58, p. 242.

28. Hilleke R.O., Chaddah P., Simmons R.O., Price D.L., Sinha S.K. // Phys. Rev. Lett., 1984, vol. 52, p. 847.

29. Sears V.F. // Phys. Rev. B, 1984, vol. 30, p. 44.

30. Watson G.I. // J. Phys.: Condens. Matter, 1996, vol. 8, p. 5955.

31. Lovesey S.W. Theory of Neutron Scattering from Condensed Matter. Oxford, Oxford University Press, 1987.

32. Peek D.A., Schmidt M.C., Fujita I., Simmons R.O. // Phys. Rev. B, 1992, vol. 45, p. 9671.

33. Peek D.A., Schmidt M.C., Fujita I., Simmons R.O. // Phys. Rev. B, 1992, vol. 45, p. 9680 .

34. Herwig K. W., Sokol P.E., Sosnick T.R., Snow W.M., Blasdell R.C. // Phys. Rev. B, 1990, vol. 41, p. 103.

35. Sosnick T.R., Snow W. M., Sokol P.E. // Phys. Rev. B, 1990, vol. 41, p. 11185.

36. Andreani C., Filabozzi A., Nardone M., Ricci F.P., Mayers J. // Phys. Rev. B, 1994, vol. 50, p. 12744.

37. Bafile U., Zoppi M., Barocchi F., Magli R., Mayers J. // Phys. Rev. Lett., 1995, vol. 75 , p. 1957.

38. Bafile U., Zoppi M., Barocchi F., Magli R., Mayers J. // Phys. Rev. B, 1996, vol. 54, p. 11969.

39. Mayers J. // Phys. Rev. Lett., 1993, vol. 71, p. 1553.

40. Andreani C., Filabozzi A., Pace E. // Phys. Rev. B, 1995, vol. 51, p. 8854.

41. Bafile U., Zoppi M., Celli M. // Physica B, 1996, vol. 226, p. 304.

42. Bafile U., Zoppi M., Celli M., Magli R., Evans A.C., Mayers J. // Physica B, 1996, vol. 217 , p. 50.

43. Bafile U., Zoppi M., Celli M., Mayers J. // Phys. Rev. B, 1998, vol. 58, p. 791. 
44. Andreani C., Filabozzi A., Pace E., Colognesi D., Zoppi M. // J. Phys.: Condens. Matter, 1998, vol. 10, p. 7091; Andreani C., Pace E., Colognesi D. // Phys. Rev. B, 1999, vol. 60, p. 10008.

45. Langel W., Price D.L., Simmons R.O., Sokol P.E. // Phys. Rev. B, 1988, vol. 38, p. 11275.

46. Celli M., Colognesi D., Zoppi M. // Eur. Phys. J. B, 2000, vol. 14, p. 239.

47. Zoppi M., Celli M., Colognesi D. // Eur. Phys. J. B, 2001, vol. 23, p. 171.

48. Zoppi M., Celli M., Colognesi D. // Europhys. Lett., 2001, vol. 53, p. 34.

49. Celli M., Colognesi D., Zoppi M. // Phys. Rev. E, 2002, vol. 66, p. 21202.

50. Van Kranendonk J. Solid Hydrogen. New York, Plenum Press, 1983.

51. Sears V.F. // Can. J. Phys., 1966, vol. 44, p. 1279.

52. Zoppi M. // Physica B, 1993, vol. 183, p. 235.

53. Young J.A., Koppel J.U. // Phys. Rev. A, 1964, vol. 33, p. 603.

54. Rahman A., Singwi K.S., Sjölander A. // Phys. Rev., 1962, vol. 126, p. 986.

55. Bermejo F.J., Kinugawa K., Cabrillo C., Bennington S.M., Fak B., FernándezDíaz M.T., Verkerk P., Dawidowski J., Fernández-Perea R. // Phys. Rev. Lett., 2000, vol. 84, p. 5359; Zoppi M., Neumann M., Celli M. // Phys. Rev. B, 2002, vol. 65, p. 092204.

56. Zoppi M., Magli R., Howells W.S., Soper A.K. // Phys. Rev. A, 1989, vol. 39, p. 4684; Zoppi M., Bafile U., Magli R., Soper A.K. // Phys. Rev. E, 1993, vol. 48, p. 1000; Zoppi M., Bafile U., Guarini E., Barocchi F., Magli R., Neumann M. // Phys. Rev. Lett., 1995, vol. 75, p. 1779; Zoppi M., Soper A.K., Barocchi F., Magli R., Bafile U., Ashcroft N.W. // Phys. Rev. E, 1996, vol. 54, p. 2773.

57. Glyde H.R. Excitations in Liquid and Solid Helium. Oxford, Clarendon Press, 1984.

58. Mahan G.D. Many-particle Physics. New York, Plenum Press, 1990.

59. Glyde H.R. // Phys. Rev. B, 1994, vol. 50, p. 6726.

60. Sears V.F. // Phys. Rev., 1969, vol. 185, p. 200.

61. Andersen K.H., Stirling W.G., Glyde H.R. // Phys. Rev. B, 1997, vol. 56, p. 8978.

62. Bowden Z.A., Celli M., Cilloco F., Colognesi D., Newport R.J., Parker S.F., Ricci F.P., Rossi-Albertini V., Sacchetti F., Tomkinson J., Zoppi M. // Physica B, 2000, vol. 98, p. $276-278$.

63. Huber K.P., Herzberg G. Constants of Diatomic Molecules. New York, van Nostrand Reinhold Company, 1979.

64. Sears V.F. // Neutron News, 1992, vol. 3, p. 29.

65. McCarty R.D., Hord J., Roder H.M. Selected Properties of Hydrogen. NBS Monograph, vol. 168, 1981.

66. Agrawal A.K. // Phys. Rev. A, 1971, vol. 4, p. 1560.

67. Celli M., Rhodes N., Soper A.K., Zoppi M. // J. Phys.: Condens. Matter, 1999, vol. 11, p. 10229.

68. James F. MINUIT Minimization Package: Reference Manual. Geneva, CERN Program Library, 1994.

69. Silvera I.F., Goldman V.V. // J. Chem. Phys., 1978, vol. 69, p. 4209.

70. Herwig K.W., Simmons R.O. // Mol. Phys., 1992, vol. 75, p. 1393.

71. Prydz R. NBS Report No. 9276, Boulder, CO, 1967 (unpublished); Roder H.M., Childs G.E., McCarty R.D., Angerhofer P.E. NBS Technical Note No. 641, 1973 (unpublished).

72. Corradi G. Determinazione dell'energia cinetica traslazionale media del deuterio liq- 
uido tramite misure di spettroscopia neutronica. Master degree thesis. University of Florence (IT), 2001 (unpublished).

73. Morishima N., Mizobuchi D. // Nucl. Instr. and Meth. A, 1994, vol. 350, p. 275; Morishima N. // Ann. Nucl. Energy, 2000, vol. 27, p. 505.

74. Neumann M., Zoppi M. // Phys. Rev. A, 1991, vol. 44, p. 2474.

75. Mompeàn F.J., García-Hernández M., Bermejo F.J., Bennington S.M. // Phys. Rev. B, 1996, vol. 54, p. 970.

76. Singwi K.S., Tosi M.P. // Phys. Rev., 1966, vol. 149, p. 70.

\title{
Вивчення середньої кінетичної енергії та залишкових ефектів в рідкому водні за допомогою непружного нейтронного розсіяння
}

\author{
Дж.Корраді ${ }^{1,2}$, Д.Колонезі ${ }^{1}$, М.Целлі ${ }^{1}$, М.Зоппі ${ }^{1}$ \\ 1 Національна Рада природничих досліджень, \\ Інститут прикладної фізики 'Нелло Каррара', \\ вул. Панціатічі 64, 50127 Флоренція, Італія \\ 2 Університет м. Флоренція, фізичний факультет, \\ вул. Г.Сансоне 1, 50019 Сесто Фіорентіна, Італія
}

Отримано 29 квітня 2003 р., в остаточному вигляді - 21 липня 2003 p.

Використовуючи спектрометр TOSCA в ISIS нами було проведено вимірювання спектрів нейтронного розсіяння рідкого пара-водню $\left(T=19.3 \mathrm{~K} \mathrm{i} n=25.4 \mathrm{~nm}^{-3}\right)$ і рідкого орто-дейтерію $(T=21.3 \mathrm{~K}$ і $n=25.2 \mathrm{~nm}^{-3}$ ). 3 високоенергетичної області спектру $40 \mathrm{meV}<$ $\hbar \omega<1 \mathrm{eV}$, де застосовується некогерентне наближення для руху центра мас, маємо можливість отримати трансляційну середню кінетичну енергію, значення якої, як ми і сподівалися, відрізняється від класичних значень. Проте, було відзначено сильні відхилення від імпульсного наближення і тому певні поправочні доданки (врахування т.зв. ефектів кінцевого стану) повинні бути взяті до розгляду для точного опису експериментальних спектрів. Порівняння наявних даних по середній кінетичній енергії з значеннями експериментальними чи з моделювань, доступними в літературі, є зовсім добрим і підтверджує високу якість процедури спектрального аналізу на спектрометрі TOSCA для конденсованих фаз рідких воднів.

Ключові слова: рідкий пара-водень, рідкий орто-дейтерій, середня кінетична енергія, ефекти кінцевого стану

PACS: $67.90 .+z, 61.12 . E x$ 
